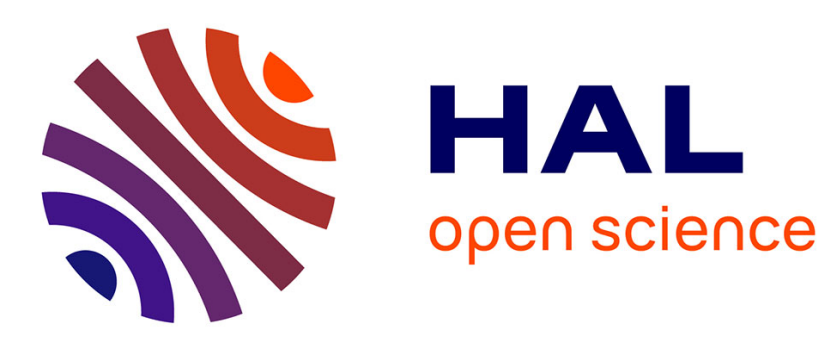

\title{
Reduced Order Modeling for Systems with Parametric Uncertainty Using Proper Generalized Decomposition
}

Parikshit Dutta

\section{To cite this version:}

Parikshit Dutta. Reduced Order Modeling for Systems with Parametric Uncertainty Using Proper Generalized Decomposition. American Control Conference, IEEE Control System Society, Jul 2015, Chicago, United States. pp.613 - 618 10.1109/ACC.2015.7170803 . hal-01132280

\section{HAL Id: hal-01132280 \\ https://hal.inria.fr/hal-01132280}

Submitted on 16 Mar 2015

HAL is a multi-disciplinary open access archive for the deposit and dissemination of scientific research documents, whether they are published or not. The documents may come from teaching and research institutions in France or abroad, or from public or private research centers.
L'archive ouverte pluridisciplinaire HAL, est destinée au dépôt et à la diffusion de documents scientifiques de niveau recherche, publiés ou non, émanant des établissements d'enseignement et de recherche français ou étrangers, des laboratoires publics ou privés. 


\title{
Reduced Order Modeling for Systems with Parametric Uncertainty Using Proper Generalized Decomposition
}

\author{
Parikshit Dutta ${ }^{1}$
}

\begin{abstract}
In this work we have proposed a new technique of model order reduction for linear time invariant (LTI) systems with parametric uncertainty. The model order reduction method is based on proper generalized decomposition (PGD). Using PGD, the underlying state variable is expanded as a sum of separated functions of time and uncertain parameters. At first, the stochastic states of the LTI system is represented using PGD. Then equations to obtain the PGD basis functions are derived. Furthermore a state feedback structure for the control input is assumed where the gain is found by solving a minimum expectation linear quadratic regulator (LQR) problem. An algorithm is then proposed, from which the PGD basis functions and the control input gain are found. The proposed algorithm is then applied to control the angle of attack and pitch rate of a F-16 aircraft having uncertain parameters. It is found that the proposed technique based on PGD could successfully achieve the control objective for the current application.
\end{abstract}

\section{INTRODUCTION}

Several real-world applications involving dynamical systems require solving sets of differential equations in high dimensional spaces. These applications range from quantum chemistry to models of financial mathematics (e.g. option pricing), and from biological systems (e.g. cell signaling) to aerospace applications (e.g. control of aircraft) [1]. Solving such problems is generally difficult and is computationally expensive. When normal discretization techniques are used these models exhibit the redoubtable curse of dimensionality [2]. The computational cost to solve these problems increases exponentially with increase in dimensions. Moreover, standard models can exhibit multidimensional behavior due to unknown parameters.

Reduced order modeling has become a popular alternative to solve multidimensional problems involving differential equations [3]. It provides an efficient way to minimize computational cost and get a sufficiently good approximation of the solution. A reduced order model creates a surrogate of the original problem using a representation of lesser dimensionality. This, in a way circumvents the curse of dimensionality associated in solving multidimensional models [4]. Over the years, researchers have developed several methods for reduced order modeling. Among them, the most popular is proper orthogonal decomposition (POD) where the underlying state variable is represented using its singular value expansion [5]. If the state variable is a stochastic process then the POD method is also known as the Karhunen Loève (KL) decomposition [6]. For systems involving parametric uncertainty one can use the polynomial chaos (PC)

\footnotetext{
${ }^{1}$ Currently with Optimal Synthesis Inc., Los Altos, CA. pdutta@optisyn.com
}

expansion [7]. However, the main issue with these methods is the evaluation of the reduced basis when the application domain is expanded. In order to obtain an sufficiently good approximation the reduced order model should be enriched and enrichment procedures still remain as an open issue [8]. Several non-intrusive methods like Monte Carlo sampling exist in the literature of reduced order modeling. However, it is well known that obtaining a solution using such nonintrusive methods is computationally expensive [9].

In this work, we have proposed a model reduction algorithm for systems with parametric uncertainty, based on proper generalized decomposition (PGD) [10]. The idea is to decompose the state variable into separate functions of deterministic variables and stochastic variables. However, PGD differs from other reduced order modeling approaches as both the functions are not fixed apriori. The optimal reduced bases are found after solving an user specified optimization problem. Researchers have used PGD to solve several problems involving parametric uncertainty. Chevreuil et al. have used PGD to propagate uncertainty in a structural dynamics problem with uncertain parameters [11]. González et al. have used PGD for parameter identification of the Cauchy's problem using data driven approaches [12]. As far as systems and control theory is concerned, Ghnatios et al. used PGD to control a thermal process where the process parameters are assumed to be uncertain [13]. The control objective in their work, is to find the optimal PGD expansion of the state variables such that the temperature reaches a prespecified value. However, designing a controller to track a specific target in presence of parametric uncertainty coupled with solving for PGD separated expansion, has not been explored in the literature.

In this work we propose a method to regulate the trajectory of a linear time invariant (LTI) dynamical system with parametric uncertainty by solving a minimum expectation linear quadratic regulator (LQR) problem. The state transition matrix is assumed to contain uncertain parameters. The state variables are represented using PGD expansion. A minimum expectation LQR problem is then solved with the LTI dynamical system as constraints. The minimum expectation problem is defined in a similar manner as in Ref. [14]. Finally the resulting control design algorithm is applied to track the trim values of a F-16 aircraft for short period dynamics with parametric uncertainty.

There are two key contributions of this paper :

1) Firstly, we are able to show how PGD can be applied to represent a LTI dynamical system with parametric uncertainty, and how the optimal PGD representation 
is obtained.

2) Secondly we have coupled the minimum expectation LQR problem with PGD represented LTI system and are able to obtain a control law which will ensure that the system reaches the desired final state.

Though the proposed method has been applied to a system with dimensionality 2 , it can be extended to systems of higher dimensions. Several examples where PGD has been applied to to reduce the order of a high dimensional system can be found in Ref. [4].

The rest of the paper is organized as follows: In Section II] we introduce how PGD is used for model reduction. In Section [II] we use PGD to expand states of the LTI dynamical system and solve for the separated representation. In section IV we use PGD to derive equations for the minimum expectation LQR problem. In Section $\mathrm{V}$ we propose an algorithm to solve the coupled system, to obtain the gain of the controller and the PGD basis functions. In Section VI we apply the PGD based methodology to control an F-16 aircraft at trim values. Finally in Section $\overline{V I I}$ we state the conclusions of the paper highlighting some future work to be done.

\section{Reduced Order Modeling Using Proper GENERALIZED DECOMPOSITION}

Let $(\Omega, \mathcal{F}, \mathbb{P})$ define an abstract probability space with $\Omega$ being the sample space and $\mathcal{F}$ being the $\sigma$-algebra of events in $\Omega, \mathbb{P}$ is the probability measure. Let us define the random vector $\boldsymbol{\Delta}:(\Omega, \mathcal{F}) \mapsto\left(\mathbb{R}^{d}, \mathcal{B}\left(\mathbb{R}^{d}\right)\right)$, where $\mathcal{B}(\cdot)$ refers to the Borel $\sigma$-algebra and $\boldsymbol{\Delta}=\left[\Delta_{1}, \ldots, \Delta_{d}\right]^{\top}$. A stochastic process in $t$ and $\boldsymbol{\Delta}$ is defined by the following map $\boldsymbol{x}(t, \boldsymbol{\Delta})$ : $[0, T] \times\left(\mathbb{R}^{d}, \mathcal{B}\left(\mathbb{R}^{d}\right)\right) \mapsto \mathbb{R}^{m}$. In PGD framework, we seek to represent $\boldsymbol{x}(t, \boldsymbol{\Delta})$ as a summation of separated functions of $t$ and $\boldsymbol{\Delta}$. A convergent approximation then can be written as,

$$
\begin{aligned}
\boldsymbol{x}(t, \boldsymbol{\Delta}) & =\sum_{i=1}^{\infty} \phi_{i}^{1}\left(\Delta_{1}\right) \ldots \phi_{i}^{d}\left(\Delta_{d}\right) \mathcal{X}_{i}(t), \\
\Rightarrow \boldsymbol{x}(t, \boldsymbol{\Delta}) & =\sum_{i=1}^{\infty} \Phi_{i}(\boldsymbol{\Delta}) \mathcal{X}_{i}(t)
\end{aligned}
$$

where $\Phi_{i}(\boldsymbol{\Delta}) \in(\mathbb{R}, \mathcal{B}(\mathbb{R}))$, and $\mathcal{X}_{i}(t) \in \mathbb{R}^{m} \forall i=1, \ldots, n$. If we truncate the sum to $N$ terms, we have,

$$
\boldsymbol{x}^{N}(t, \boldsymbol{\Delta})=\sum_{i=1}^{N} \Phi_{i}(\boldsymbol{\Delta}) \mathcal{X}_{i}(t),
$$

In PGD framework, the sum $\boldsymbol{x}^{N}(t, \boldsymbol{\Delta})$ is constructed incrementally by solving for the functions $\Phi_{i}(\boldsymbol{\Delta})$ and $X_{i}(t)$. We assume that the sum given in (2) is known upto $n<N$ terms. Next we introduce a candidate $n+1^{\text {th }}$ term such that

$$
\boldsymbol{x}^{n+1}(t, \boldsymbol{\Delta})=\boldsymbol{x}^{n}(t, \boldsymbol{\Delta})+P(\boldsymbol{\Delta}) \boldsymbol{Y}(t) .
$$

where $P(\boldsymbol{\Delta}) \in(\mathbb{R}, \mathcal{B}(\mathbb{R}))$ and $\boldsymbol{Y}(t) \in \mathbb{R}^{m}$ are unknown functions. The couple $P(\boldsymbol{\Delta})$ and $\boldsymbol{Y}(t)$ is known as the enrichment couple. We then solve for $P(\boldsymbol{\Delta})$ and $\boldsymbol{Y}(t)$. This enrichment procedure continues until,

$$
\left\|\boldsymbol{x}^{n+1}(t, \boldsymbol{\Delta})-\boldsymbol{x}^{n}(t, \boldsymbol{\Delta})\right\|<\epsilon
$$

where $\epsilon$ is a user specified number determining convergence and $\|\cdot\|$ is appropriate norm. If the enrichment procedure stops at $N<\infty$ the resulting approximation $\boldsymbol{x}^{N}(t, \boldsymbol{\Delta})$ is of rank $N$ rather than the $\infty$ rank of the actual random process.

In this work we have discussed the PGD construction algorithm when the governing equation is a LTI system. For detailed information about PGD basis function construction, interested readers are directed to Ref. [15].

\section{Proper Generalized Decomposition of Linear TIME INVARIANT SYSTEMS}

We consider the linear system, whose dynamics is given by,

$$
\dot{\boldsymbol{x}}(t, \boldsymbol{\Delta})=\boldsymbol{A}(\boldsymbol{\Delta}) \boldsymbol{x}(t, \boldsymbol{\Delta})+\boldsymbol{B} \boldsymbol{u}(t, \boldsymbol{\Delta})
$$

where, $\boldsymbol{x}(t, \boldsymbol{\Delta}) \in\left(\mathbb{R}^{m}, \mathcal{B}\left(\mathbb{R}^{m}\right)\right)$ are the states and $\boldsymbol{u}(t, \boldsymbol{\Delta}) \in$ $\left(\mathbb{R}^{p}, \mathcal{B}\left(\mathbb{R}^{p}\right)\right)$ are the control inputs. $\boldsymbol{\Delta}$ is a $\mathbb{R}^{d}$-valued vector of random variables and $\left\{\Delta_{i}\right\}_{i=1}^{d} \in(\mathbb{R}, \mathcal{B}(\mathbb{R})) . \boldsymbol{A}(\Delta) \in$ $\left(\mathbb{R}^{m \times m}, \mathcal{B}\left(\mathbb{R}^{m \times m}\right)\right)$ and $\boldsymbol{B} \in \mathbb{R}^{m \times p}$ are assumed to be given.

Substituting the incremental representation given in (3), in (4), we have,

$$
\begin{gathered}
\dot{\boldsymbol{x}}^{n}(t, \boldsymbol{\Delta})+P(\boldsymbol{\Delta}) \dot{\boldsymbol{Y}}(t)=\boldsymbol{A}(\boldsymbol{\Delta})\left[\boldsymbol{x}^{n}(t, \boldsymbol{\Delta})+\right. \\
P(\boldsymbol{\Delta}) \boldsymbol{Y}(t)]+\boldsymbol{B} \boldsymbol{u}(t, \boldsymbol{\Delta}), \\
\Rightarrow P(\boldsymbol{\Delta}) \dot{\boldsymbol{Y}}(t)=\boldsymbol{A}(\boldsymbol{\Delta}) P(\boldsymbol{\Delta}) \boldsymbol{Y}(t)+\boldsymbol{B} \boldsymbol{u}(t, \boldsymbol{\Delta}) \\
-\left[\dot{x}^{n}(t, \boldsymbol{\Delta})-\boldsymbol{A}(\boldsymbol{\Delta}) \boldsymbol{x}^{n}(t, \boldsymbol{\Delta})\right] .
\end{gathered}
$$

For the control $\boldsymbol{u}(t, \boldsymbol{\Delta})$, we assume a state feedback form with gain calculated appropriately. In this work, we have used a constant gain $\boldsymbol{K}$ such that $\boldsymbol{u}(t, \boldsymbol{\Delta})=-\boldsymbol{K} \boldsymbol{x}(t, \boldsymbol{\Delta})$. Assuming other forms of gain is possible and is a subject of our future work. Substituting $\boldsymbol{u}(t, \boldsymbol{\Delta})$ in $(5)$, we have,

$$
\begin{aligned}
& P(\boldsymbol{\Delta}) \dot{\boldsymbol{Y}}(t)=\boldsymbol{A}(\boldsymbol{\Delta}) P(\boldsymbol{\Delta}) \boldsymbol{Y}(t)-\boldsymbol{B} \boldsymbol{K} P(\boldsymbol{\Delta}) \boldsymbol{Y}(t) \\
& \quad-\left[\dot{x}^{n}(t, \boldsymbol{\Delta})-\boldsymbol{A}(\boldsymbol{\Delta}) \boldsymbol{x}^{n}(t, \boldsymbol{\Delta})+\boldsymbol{B} \boldsymbol{K} \boldsymbol{x}^{n}(t, \boldsymbol{\Delta})\right](6)
\end{aligned}
$$

In the above equation, $P(\boldsymbol{\Delta}), \boldsymbol{Y}(t)$ and $\boldsymbol{K}$ are unknowns. Due to incremental nature of the PGD construction algorithm, $\boldsymbol{x}^{n}(t, \boldsymbol{\Delta})$ is known.

To find $P(\boldsymbol{\Delta})$ and $\boldsymbol{Y}(t)$, we use the weak integral form of the ODE in (4). First we assume small perturbation in $\boldsymbol{x}^{n}(t, \boldsymbol{\Delta})$ as $\delta \boldsymbol{x}^{n}(t, \boldsymbol{\Delta})$, which acts as the test function. The integral form is then written as,

$$
\begin{aligned}
\int_{0}^{T} \int_{\boldsymbol{\Omega}} \delta \boldsymbol{x}^{n}(t, \boldsymbol{\Delta}) & {\left[\dot{\boldsymbol{x}}^{n+1}(t, \boldsymbol{\Delta})-\boldsymbol{A}(\boldsymbol{\Delta}) \boldsymbol{x}^{n+1}(t, \boldsymbol{\Delta})\right.} \\
& \left.+\boldsymbol{B} \boldsymbol{K} \boldsymbol{x}^{n+1}(t, \boldsymbol{\Delta})\right] d t f(\boldsymbol{\Delta}) d \boldsymbol{\Delta}=0,
\end{aligned}
$$

where $t \in[0, T]$ and $\boldsymbol{\Delta} \in \boldsymbol{\Omega} \subset \mathbb{R}^{d}, f(\boldsymbol{\Delta})$ is the probability density function (PDF) of $\boldsymbol{\Delta}$. The test function $\delta \boldsymbol{x}^{n}(t, \boldsymbol{\Delta})$ is considered to be a function of the enrichment couple, $P(\boldsymbol{\Delta})$ and $\boldsymbol{Y}(t)$, which is given by,

$$
\delta \boldsymbol{x}^{n}(t, \boldsymbol{\Delta})=P(\boldsymbol{\Delta}) \boldsymbol{\delta} Y(t)+\boldsymbol{Y}(t) \delta P(\boldsymbol{\Delta})
$$

where $\delta \boldsymbol{Y}(t)$ and $\delta P(\boldsymbol{\Delta})$ are perturbations in $\boldsymbol{Y}(t)$ and $P(\boldsymbol{\Delta})$, respectively. Using the test function in 8 in 6 , 
we have,

$$
\begin{aligned}
& \int_{0}^{T} \int_{\boldsymbol{\Omega}}[P(\boldsymbol{\Delta}) \delta \boldsymbol{Y}(t)+\boldsymbol{Y}(t) \delta P(\boldsymbol{\Delta})]^{\top}[P(\boldsymbol{\Delta}) \dot{\boldsymbol{Y}}(t)-\boldsymbol{A}(\boldsymbol{\Delta}) P(\boldsymbol{\Delta}) \boldsymbol{Y}(t) \\
& +\boldsymbol{B} \boldsymbol{K} P(\boldsymbol{\Delta}) \boldsymbol{Y}(t) \\
& +\underbrace{\left\{\dot{x}^{n}(t, \boldsymbol{\Delta})-\boldsymbol{A}(\boldsymbol{\Delta}) \boldsymbol{x}^{n}(t, \boldsymbol{\Delta})+\boldsymbol{B} \boldsymbol{K} \boldsymbol{x}^{n}(t, \boldsymbol{\Delta})\right\}}_{\boldsymbol{F}^{n}(t, \boldsymbol{\Delta})}] d t f(\boldsymbol{\Delta}) d \boldsymbol{\Delta}=0 \quad(9)
\end{aligned}
$$

where $\boldsymbol{F}^{n}(t, \boldsymbol{\Delta})$ and can be integrated w.r.t. $t$ and $\boldsymbol{\Delta}$. Further simplifying (9), we have,

$$
\begin{gathered}
\int_{0}^{T} \int_{\boldsymbol{\Omega}}[P(\boldsymbol{\Delta}) \delta \boldsymbol{Y}(t)]^{\top}[P(\boldsymbol{\Delta}) \dot{\boldsymbol{Y}}(t)-\boldsymbol{A}(\boldsymbol{\Delta}) P(\boldsymbol{\Delta}) \boldsymbol{Y}(t) \\
\left.+\boldsymbol{B} \boldsymbol{K} P(\boldsymbol{\Delta}) \boldsymbol{Y}(t)+\boldsymbol{F}^{n}(t, \boldsymbol{\Delta})\right] d t f(\boldsymbol{\Delta}) d \boldsymbol{\Delta}+ \\
\int_{0}^{T} \int_{\boldsymbol{\Omega}}[\boldsymbol{Y}(t) \delta P(\boldsymbol{\Delta})]^{\top}[P(\boldsymbol{\Delta}) \dot{\boldsymbol{Y}}(t)-\boldsymbol{A}(\boldsymbol{\Delta}) P(\boldsymbol{\Delta}) \boldsymbol{Y}(t) \\
\left.+\boldsymbol{B} \boldsymbol{K} P(\boldsymbol{\Delta}) \boldsymbol{Y}(t)+\boldsymbol{F}^{n}(t, \boldsymbol{\Delta})\right] d t f(\boldsymbol{\Delta}) d \boldsymbol{\Delta}=0
\end{gathered}
$$

\section{A. Computing the function $\boldsymbol{Y}(t)$}

To compute $\boldsymbol{Y}(t)$ we assume that $P(\boldsymbol{\Delta})$ is known. hence $\delta P(\boldsymbol{\Delta})$ vanishes). Also noting the fact that $P(\boldsymbol{\Delta})$ is a scalar, [10] is then given by,

$$
\begin{aligned}
\int_{0}^{T} \int_{\boldsymbol{\Omega}} \delta \boldsymbol{Y}(t)^{\top} P(\boldsymbol{\Delta})[P(\boldsymbol{\Delta}) \dot{\boldsymbol{Y}}(t)-\boldsymbol{A}(\boldsymbol{\Delta}) P(\boldsymbol{\Delta}) \boldsymbol{Y}(t) \\
\left.+\boldsymbol{B} \boldsymbol{K} P(\boldsymbol{\Delta}) \boldsymbol{Y}(t)+\boldsymbol{F}^{n}(t, \boldsymbol{\Delta})\right] d t f(\boldsymbol{\Delta}) d \boldsymbol{\Delta}=0
\end{aligned}
$$

The above equation can be written as,

$$
\begin{aligned}
& \int_{0}^{T} \delta \boldsymbol{Y}(t)\left[\dot{\boldsymbol{Y}}(t) \int_{\boldsymbol{\Omega}} P^{2}(\boldsymbol{\Delta}) f(\boldsymbol{\Delta}) d \boldsymbol{\Delta}-\left(\int_{\boldsymbol{\Omega}} P^{2}(\boldsymbol{\Delta}) \boldsymbol{A}(\boldsymbol{\Delta}) f(\boldsymbol{\Delta}) d \boldsymbol{\Delta}\right) \boldsymbol{Y}(t)\right. \\
& \left.+\boldsymbol{B} \boldsymbol{K} \boldsymbol{Y}(t) \int_{\boldsymbol{\Omega}} P^{2}(\boldsymbol{\Delta}) f(\boldsymbol{\Delta}) d \boldsymbol{\Delta}+\int_{\boldsymbol{\Omega}} P(\boldsymbol{\Delta}) \boldsymbol{F}^{n}(t, \boldsymbol{\Delta}) f(\boldsymbol{\Delta}) d \boldsymbol{\Delta}\right] d t=0
\end{aligned}
$$

The above equation is true for all perturbations $\delta \boldsymbol{Y}(t)$, hence we have

$$
P_{1} \dot{\boldsymbol{Y}}(t)-\boldsymbol{P}_{\mathbf{2}} \boldsymbol{Y}(t)+P_{1} \boldsymbol{B} \boldsymbol{K} \boldsymbol{Y}(t)+\boldsymbol{P}_{3}(t)=0,
$$

where,

$$
\begin{aligned}
& P_{1}=\int_{\boldsymbol{\Omega}} P^{2}(\boldsymbol{\Delta}) f(\boldsymbol{\Delta}) d \boldsymbol{\Delta}, \boldsymbol{P}_{\mathbf{2}}=\int_{\boldsymbol{\Omega}} P^{2}(\boldsymbol{\Delta}) \boldsymbol{A}(\boldsymbol{\Delta}) f(\boldsymbol{\Delta}) d \boldsymbol{\Delta}, \\
& \boldsymbol{P}_{3}(t)=\int_{\boldsymbol{\Omega}} P(\boldsymbol{\Delta}) \boldsymbol{F}^{n}(t, \boldsymbol{\Delta}) f(\boldsymbol{\Delta}) d \boldsymbol{\Delta} .
\end{aligned}
$$

$P_{1} \in \mathbb{R}$ is a scalar constant, $\boldsymbol{P}_{\mathbf{2}} \in \mathbb{R}^{m \times m}$ is a constant matrix. $\boldsymbol{P}_{3} \in \mathbb{R}^{m}$ is a known function of $t$. Eqn. (13) is a nonlinear ODE in $\boldsymbol{Y}(t)$, which can be solved using appropriate ODE solvers [2].

\section{B. Computing the function $P(\boldsymbol{\Delta})$}

To compute $P(\boldsymbol{\Delta}), \boldsymbol{Y}(t)$ is assumed to be known, hence the perturbation $\delta \boldsymbol{Y}(t)$ vanishes. Hence 10 can be written as,

$$
\begin{array}{r}
\int_{0}^{T} \int_{\boldsymbol{\Omega}} \delta P(\boldsymbol{\Delta})^{\top} \boldsymbol{Y}(t)^{\top}[P(\boldsymbol{\Delta}) \dot{\boldsymbol{Y}}(t)-\boldsymbol{A}(\boldsymbol{\Delta}) P(\boldsymbol{\Delta}) \boldsymbol{Y}(t) \\
\left.+\boldsymbol{B} \boldsymbol{K} P(\boldsymbol{\Delta}) \boldsymbol{Y}(t)+\boldsymbol{F}^{n}(t, \boldsymbol{\Delta})\right] d t f(\boldsymbol{\Delta}) d \boldsymbol{\Delta}=0
\end{array}
$$

Eqn. (14) can be simplified as,

$$
\begin{aligned}
& \int_{\boldsymbol{\Omega}} \delta P(\boldsymbol{\Delta})\left[P(\boldsymbol{\Delta}) \int_{0}^{T} \mathbf{Y}(t)^{\top} \dot{\boldsymbol{Y}}(t) d t-P(\boldsymbol{\Delta}) \int_{0}^{T} \mathbf{Y}(t)^{\top} \boldsymbol{A}(\boldsymbol{\Delta}) Y(t) d t\right. \\
& \left.+P(\boldsymbol{\Delta}) \int_{0}^{T} \boldsymbol{Y}(t)^{\top} \boldsymbol{B} \boldsymbol{K} Y(t) d t+\int_{0}^{T} \boldsymbol{Y}(t)^{\top} \boldsymbol{F}^{n}(t, \boldsymbol{\Delta}) d t\right] f(\boldsymbol{\Delta}) d \boldsymbol{\Delta}=0
\end{aligned}
$$

As the above equations is true for all $\delta P(\boldsymbol{\Delta})$, hence we have,

$$
Y_{1} P(\boldsymbol{\Delta})-Y_{2}(\boldsymbol{\Delta}) P(\boldsymbol{\Delta})+Y_{3} P(\boldsymbol{\Delta})+Y_{4}(\boldsymbol{\Delta})=0,
$$

where

$Y_{1}=\int_{0}^{T} \boldsymbol{Y}(t)^{\top} \dot{\boldsymbol{Y}}(t) d t, Y_{2}(\Delta)=\sum_{i=1}^{m} \sum_{j=1}^{m} a_{i j}(\boldsymbol{\Delta}) \int_{0}^{T} y_{i}(t) y_{j}(t) d t$,

$Y_{3}=\sum_{i=1}^{m} \sum_{j=1}^{m} \sum_{l=1}^{p} b_{i l} k_{l j} \int_{0}^{T} y_{i}(t) y_{j}(t) d t, Y_{4}(\Delta)=\int_{0}^{T} \boldsymbol{Y}(t)^{\top} \boldsymbol{F}^{n}(t, \boldsymbol{\Delta}) d t$,

where, $\boldsymbol{A}(\boldsymbol{\Delta})=\left[a_{i j}(\boldsymbol{\Delta})\right], i, j=1, \ldots, m, \boldsymbol{B}=\left[b_{i j}\right], i=$ $1, \ldots, m, j=1, \ldots, p, \boldsymbol{K}=\left[k_{i j}\right], i=1, \ldots, p, j=$ $1, \ldots, m$ and $\boldsymbol{Y}(t)=\left[y_{1}(t), \ldots, y_{m}(t)\right]^{\top} . Y_{1}$ and $Y_{3}$ are constant scalars and $Y_{2}(\boldsymbol{\Delta})$ and $Y_{4}(\boldsymbol{\Delta})$ are known functions of $\boldsymbol{\Delta}$. Eqn. (16) is an algebraic equation in $P(\boldsymbol{\Delta})$ from where $P(\boldsymbol{\Delta})$ can be obtained.

\section{Linear QuAdratic Regulator USING PRoper GENERALIZED DECOMPOSITION}

Eqn. (13) and (16) are ODE and algebraic equation in $\boldsymbol{Y}(t)$ and $P(\boldsymbol{\Delta})$, respectively from where the enrichment couple $P(\boldsymbol{\Delta}) \boldsymbol{Y}(t)$ can be obtained. To this end, a methodology to construct a separated representation for the unknown states $\boldsymbol{x}^{n}(t, \Delta)$ incrementally has been presented. However, in the method described in Section III] we have not specified how to calculate the gain $\boldsymbol{K}$. The gain $\boldsymbol{K}$ is generally obtained by solving a control law design problem based on the control objectives specified by the user. In this work, we have solved an finite time, linear quadratic regulator (LQR) optimal control problem in PGD framework, to find the gain $\boldsymbol{K}$.

The finite time LQR objective function can be framed as a minimum expected cost problem as,

$$
\begin{aligned}
& J=\min _{\boldsymbol{u}} \int_{0}^{T} \mathbb{E}\left[\boldsymbol{x}(t, \boldsymbol{\Delta})^{\top} \boldsymbol{Q} \boldsymbol{x}(t, \boldsymbol{\Delta})+\boldsymbol{u}(t, \boldsymbol{\Delta})^{\top} \boldsymbol{R} \boldsymbol{u}(t, \boldsymbol{\Delta})\right] d t \\
& \text { subject to, } \\
& \qquad \dot{\boldsymbol{x}}(t, \boldsymbol{\Delta})=\boldsymbol{A}(\boldsymbol{\Delta}) \boldsymbol{x}(t, \boldsymbol{\Delta})+\boldsymbol{B} \boldsymbol{u}(t, \boldsymbol{\Delta}) .
\end{aligned}
$$

Here we have assumed stochastic state feedback with constant gain given by,

$$
\boldsymbol{u}(t, \boldsymbol{\Delta})=-\boldsymbol{K} \boldsymbol{x}(t, \boldsymbol{\Delta})
$$

We will now develop iterative equations to solve for the gain $\boldsymbol{K}$ once the enrichment couple in (13) and (16) has been determined. We will assume that we know the PGD separated expansion of $\boldsymbol{x}(t, \boldsymbol{\Delta})$ upto $n$-terms. The $\Phi_{i}(\boldsymbol{\Delta})$ are further assumed to be orthonormal to each other, hence $\int_{\boldsymbol{\Omega}} \Phi_{i}(\boldsymbol{\Delta}) \Phi_{j}(\boldsymbol{\Delta}) f(\boldsymbol{\Delta}) d \boldsymbol{\Delta}=\delta_{i j}$

It is easy to see that $n$-term approximated state in (2) can also be written as [16],

$$
\boldsymbol{x}^{n}(t, \boldsymbol{\Delta})=\left[\mathbb{I}_{m} \otimes \boldsymbol{\Phi}^{\top}(\boldsymbol{\Delta})\right] \boldsymbol{X}(t),
$$

where $\mathbb{I}_{m}$ is the identity matrix of size $m \times m, \boldsymbol{\Phi}(\boldsymbol{\Delta})=$ $\left[\Phi_{1}(\boldsymbol{\Delta}), \ldots, \Phi_{N}(\boldsymbol{\Delta})\right]^{\top}$, and $\boldsymbol{X}(t)=\left[\mathcal{X}_{1}^{\top}, \ldots, \mathcal{X}_{N}^{\top}\right]^{\top}$.

Proposition 1: Assuming the feedback structure in (18), the dynamical system in (4) can be written as,

$$
\dot{\boldsymbol{X}}(t)=\mathcal{A} \boldsymbol{X}(t)-\mathcal{B} \mathcal{K} \boldsymbol{X}(t)
$$

where $\mathcal{A}=\int_{\boldsymbol{\Omega}}[\boldsymbol{A}(\boldsymbol{\Delta}) \otimes \boldsymbol{\Phi}(\boldsymbol{\Delta}) \boldsymbol{\Phi}(\boldsymbol{\Delta})] f(\boldsymbol{\Delta}) d \boldsymbol{\Delta}, \boldsymbol{\mathcal { B }}=\boldsymbol{B} \otimes \mathbb{I}_{n}$ and $\mathcal{K}=\boldsymbol{K} \otimes I_{n}$ 
Proof: In the proof, we will drop the parenthesis of $\boldsymbol{A}(\boldsymbol{\Delta})$ and $\boldsymbol{\Phi}(\boldsymbol{\Delta})$ for simplicity in representation.

Substituting (19) in (4) and assuming stochastic state feedback as given in (18), we have

$$
\left[\mathbb{I}_{m} \otimes \boldsymbol{\Phi}^{\top}\right] \dot{\boldsymbol{X}}(t)=\boldsymbol{A}\left[\mathbb{I}_{m} \otimes \Phi^{\top}\right] \boldsymbol{X}(t)-\boldsymbol{B} \boldsymbol{K}\left[\mathbb{I}_{m} \otimes \Phi^{\top}\right] \boldsymbol{X}(t)
$$

Using Galerkin's projection rule and orthonormality criterion and properties of Kronecker product, we have,

$$
\begin{aligned}
\int_{\boldsymbol{\Omega}}\left[\mathbb{I}_{m} \otimes \boldsymbol{\Phi} \boldsymbol{\Phi}^{\top}\right] \dot{\boldsymbol{X}}(t) f(\boldsymbol{\Delta}) d \boldsymbol{\Delta}=\int_{\boldsymbol{\Omega}}\left[\boldsymbol{A} \otimes \boldsymbol{\Phi}^{\top}\right] \boldsymbol{X}(t) f(\boldsymbol{\Delta}) d \boldsymbol{\Delta} \\
-\int_{\boldsymbol{\Omega}}\left[\boldsymbol{B} \boldsymbol{K} \otimes \boldsymbol{\Phi} \boldsymbol{\Phi}^{\top}\right] \boldsymbol{X}(t) f(\boldsymbol{\Delta}) d \boldsymbol{\Delta}, \\
\Rightarrow\left[\mathbb{I}_{m} \otimes \int_{\boldsymbol{\Omega}} \boldsymbol{\Phi} \boldsymbol{\Phi}^{\top} f(\boldsymbol{\Delta}) d \boldsymbol{\Delta}\right] \dot{\boldsymbol{X}}(t)=\left[\int_{\boldsymbol{\Omega}} \boldsymbol{A} \otimes \boldsymbol{\Phi} \boldsymbol{\Phi}^{\top} f(\boldsymbol{\Delta}) d \boldsymbol{\Delta}\right] \boldsymbol{X}(t) \\
-\left[\boldsymbol{B} \boldsymbol{K} \otimes \int_{\boldsymbol{\Omega}} \boldsymbol{\Phi} \boldsymbol{\Phi}^{\top} f(\boldsymbol{\Delta}) d \boldsymbol{\Delta}\right] \boldsymbol{X}(t) \\
\Rightarrow\left[\mathbb{I}_{m} \otimes \mathbb{I}_{n}\right] \dot{\boldsymbol{X}}(t)=\underbrace{\left[\int_{\boldsymbol{A}} \boldsymbol{A} \otimes \boldsymbol{\Phi}^{\top} f(\boldsymbol{\Delta}) d \boldsymbol{\Delta}\right]}_{\boldsymbol{\Omega}} \boldsymbol{X}(t) \\
-\underbrace{\left[\boldsymbol{B} \otimes \mathbb{I}_{n}\right]}_{\boldsymbol{\mathcal { B }}} \underbrace{\left[\boldsymbol{K} \otimes \mathbb{I}_{n}\right]}_{\boldsymbol{\mathcal { K }}} \boldsymbol{X}(t)
\end{aligned}
$$

Eqn. 21] can be written as

$$
\dot{\boldsymbol{X}}(t)=\mathcal{A} \boldsymbol{X}(t)-\mathcal{B} \mathcal{K} \boldsymbol{X}(t)
$$

Proposition 2: Assuming the feedback structure in (18), the objective function in (17) can be written as,

$$
J=\min _{\boldsymbol{K}} \int_{0}^{T}\left[\boldsymbol{X}(t)^{\top} \mathcal{Q} \boldsymbol{X}(t)+\boldsymbol{X}(t)^{\top} \mathcal{K}^{\top} \mathcal{R} \mathcal{K} \boldsymbol{X}(t)\right] d t
$$

where $\mathcal{Q}=\left[\boldsymbol{Q} \otimes \mathbb{I}_{n}\right]$ and $\mathcal{R}=\left[\boldsymbol{R} \otimes \mathbb{I}_{n}\right]$

Proof: We will drop out the parenthesis of $\boldsymbol{\Phi}(\boldsymbol{\Delta})$ for simplicity in representation.

Introducing the expression in (19) and using the definition of $\mathbb{E}[\cdot]$ in the objective function of $(17)$, we have,

$$
\begin{aligned}
J= & \min _{\boldsymbol{K}} \int_{0}^{T}\left[\int _ { \boldsymbol { \Omega } } \left\{\boldsymbol{X}(t)^{\top}\left[\mathbb{I}_{m} \otimes \boldsymbol{\Phi}^{\top}\right]^{\top} \boldsymbol{Q}\left[\mathbb{I}_{m} \otimes \boldsymbol{\Phi}^{\top}\right] \boldsymbol{X}(t)\right.\right. \\
& \left.\left.+\boldsymbol{X}(t)^{\top}\left[\mathbb{I}_{m} \otimes \boldsymbol{\Phi}^{\top}\right]^{\top} \boldsymbol{K}^{\top} \boldsymbol{R} \boldsymbol{K}\left[\mathbb{I}_{m} \otimes \boldsymbol{\Phi}^{\top}\right] \boldsymbol{X}(t)\right\} f(\boldsymbol{\Delta}) d \boldsymbol{\Delta}\right] d t \\
J= & \min _{\boldsymbol{K}} \int_{0}^{T} \bar{J} d t .
\end{aligned}
$$

Expanding $\bar{J}$ and using properties of Kronecker product and orthonormality of $\boldsymbol{\Phi}$, we have, we have,

$$
\begin{aligned}
\bar{J}= & \int_{\boldsymbol{\Omega}}\left[\boldsymbol{X}(t)^{\top}\left[\mathbb{I}_{m} \otimes \boldsymbol{\Phi}^{\top}\right]^{\top} \boldsymbol{Q}\left[\mathbb{I}_{m} \otimes \boldsymbol{\Phi}^{\top}\right] \boldsymbol{X}(t)\right. \\
+ & \left.\boldsymbol{X}(t)^{\top}\left[\mathbb{I}_{m} \otimes \boldsymbol{\Phi}^{\top}\right]^{\top} \boldsymbol{K}^{\top} \boldsymbol{R} \boldsymbol{K}\left[\mathbb{I}_{m} \otimes \boldsymbol{\Phi}^{\top}\right] \boldsymbol{X}(t)\right] f(\boldsymbol{\Delta}) d \boldsymbol{\Delta} \\
= & \int_{\boldsymbol{\Omega}}\left[\boldsymbol{X}(t)^{\top}\left[\boldsymbol{Q} \otimes \boldsymbol{\Phi} \boldsymbol{\Phi}^{\top}\right] \boldsymbol{X}(t)\right. \\
& \left.\quad+\boldsymbol{X}(t)^{\top}\left[\boldsymbol{K}^{\top} \boldsymbol{R} \boldsymbol{K} \otimes \boldsymbol{\Phi} \boldsymbol{\Phi}^{\top}\right] \boldsymbol{X}(t)\right] f(\boldsymbol{\Delta}) d \boldsymbol{\Delta} \\
= & \boldsymbol{X}(t)^{\top}\left[\boldsymbol{Q} \otimes \int_{\boldsymbol{\Omega}} \boldsymbol{\Phi} \boldsymbol{\Phi}^{\top} f(\boldsymbol{\Delta}) d \boldsymbol{\Delta}\right] \boldsymbol{X}(t) \\
& \quad+\boldsymbol{X}(t)^{\top}\left[\boldsymbol{K}^{\top} \boldsymbol{R} \boldsymbol{K} \otimes \int_{\boldsymbol{\Omega}} \boldsymbol{\Phi} \boldsymbol{\Phi}^{\top} f(\boldsymbol{\Delta}) d \boldsymbol{\Delta}\right] \boldsymbol{X}(t), \\
= & \boldsymbol{X}(t)^{\top}\left[\boldsymbol{Q} \otimes \mathbb{I}_{n}\right] \boldsymbol{X}(t)+\boldsymbol{X}(t)^{\top}\left[\boldsymbol{K}^{\top} \boldsymbol{R} \boldsymbol{K} \otimes \mathbb{I}_{n}\right] \boldsymbol{X}(t), \\
= & \boldsymbol{X}(t)^{\top}\left[\boldsymbol{Q} \otimes \mathbb{I}_{n}\right] \boldsymbol{X}(t)+\boldsymbol{X}(t)^{\top}\left[\boldsymbol{K} \otimes \mathbb{I}_{n}\right]^{\top}\left[\boldsymbol{R} \otimes \mathbb{I}_{n}\right]\left[\boldsymbol{K} \otimes \mathbb{I}_{n}\right] \boldsymbol{X}(t)
\end{aligned}
$$

Hence the objective function $J$ can be written as

$$
\begin{aligned}
J=\min _{\boldsymbol{K}} \int_{0}^{T}\left[\boldsymbol{X}(t)^{\top}\left[\boldsymbol{Q} \otimes \mathbb{I}_{n}\right] \boldsymbol{X}(t)\right. \\
\left.+\boldsymbol{X}(t)^{\top}\left[\boldsymbol{K} \otimes \mathbb{I}_{n}\right]^{\top}\left[\boldsymbol{R} \otimes \mathbb{I}_{n}\right]\left[\boldsymbol{K} \otimes \mathbb{I}_{n}\right] \boldsymbol{X}(t)\right] d t
\end{aligned}
$$

From Propositions 1 and 2, it is evident that for the dynamical system in 20, the corresponding LQR objective function is given by 22. It is well known that the gain $\mathcal{K}$ is can be written as,

$$
\mathcal{K}=\mathcal{R}^{-1} \mathcal{B}^{\top} \mathcal{S}
$$

where the matrix $S$ is found by solving the Ricatti ODE given by [17],

$$
\dot{\mathcal{S}}+\mathcal{A}^{\top} \mathcal{S}+\mathcal{S} \mathcal{A}-\mathcal{S} \mathcal{B} \mathcal{R}^{-1} \mathcal{B}^{\top} \mathcal{S}+\mathcal{Q}=0, \quad \mathcal{S}=\mathcal{S}^{\top}
$$

Once $\mathcal{K}$ has been determined, one can find $\boldsymbol{K}$ using the matrix equation,

$$
\left[\boldsymbol{K} \otimes \mathbb{I}_{n}\right]=\mathcal{K}
$$

The matrix equation in 27) is over determined, the solutions of which can be found using ordinary least squares.

\section{Solution Methodology}

To obtain the separated representation, first $\boldsymbol{Y}(t)$ and $P(\boldsymbol{\Delta})$ are obtained by solving the system of equations 13 and (16), and then $\boldsymbol{K}$ is obtained from (26) and 25). Several algorithms exist in the literature to construct the PGD system iteratively [18]. However, in the current framework a constraint of orthonormality of $\Phi(\boldsymbol{\Delta})$ s should be imposed, in order to obtain the results proposed in Section IV In this work, we have proposed an algorithm using stable version of Gram-Schmidt (GS) orthonomalization to sequentially construct the summation in 22 [19]. The algorithm proposed here has been developed in a similar manner to the Arnoldi iteration with full update algorithm proposed by Nouy [18]. The algorithm is presented in Algorithm 1

As seen in the algorithm, the enrichment couple is found iteratively by solving (13) and (16) iteratively until convergence which is dictated by the metric $e_{e n}$. Furthermore once the convergence is achieved, the $P(\boldsymbol{\Delta})$ s are added to already existing set of $\Phi(\boldsymbol{\Delta})$ after Gram-Schmidt orthonormalization has been carried out [20]. The gain value $\boldsymbol{K}$ is obtained from the orthonormalized $\boldsymbol{\Phi}(\boldsymbol{\Delta})$ basis. The algorithm is terminated when the convergence metric $e$ is below a certain threshold $E_{t h}$.

\section{NUMERICAL EXAMPLES}

In this section we have applied the proposed PGD based reduced order modeling technique for minimum expectation control of a F-16 aircraft. Here we consider the short period dynamics of a F-16 aircraft, with states as the angle of attack $\alpha$ and the pitch rate $q$. We have used the linearized dynamical system, trimmed at an airspeed of $502 \mathrm{ft} / \mathrm{s}$ and angle of attack $\alpha=2.11^{\circ}$. The dynamics used here is the same as used in Ref. [21], which is given by,

$$
\begin{aligned}
{\left[\begin{array}{c}
\dot{\alpha}(\Delta, t) \\
\dot{q}(\Delta, t)
\end{array}\right]=} & {\left[\begin{array}{cc}
-1.0190 & 1 \\
\Delta & -1.0744
\end{array}\right]\left[\begin{array}{c}
\alpha(\Delta, t) \\
q(\Delta, t)
\end{array}\right] } \\
& +\left[\begin{array}{c}
0 \\
-0.1756
\end{array}\right] u(t) .
\end{aligned}
$$

The parameter $\Delta$ is assumed to be uniformly distributed with the nominal value of 0.8223 and a standard deviation 




of $\pm 20 \%$ about the nominal value. The uncertainty here is assumed to arise from the pitch damping term being unknown. The objective of the controller design is to maintain the aircraft at the trimmed values, given the uncertainty in parameters. To achieve this objective we solve a minimum expectation LQR problem which is completely described by (17). The $\boldsymbol{Q}$ and $\boldsymbol{R}$ matrices are given by,

$$
\boldsymbol{Q}=\left[\begin{array}{cc}
8 & 0 \\
0 & 0.5
\end{array}\right], \quad \boldsymbol{R}=0.01
$$

The initial states are assumed to be known and are given by $\alpha(0, \Delta), q(0, \Delta)=\left[2.11^{\circ}, 0\right]^{\top}$, for all values of $\Delta$.

We use the proposed methodology based on PGD to obtain a separated representation for the states, as well as the gain $\boldsymbol{K}$. The $E_{t h}$ in Algorithm 1 is assumed to be $10^{-4}$. The errors $e$ and $e_{e n}$ are initialized to a very high number. After solving the minimum expectation LQR using PGD method, the number of terms obtained in the PGD expansion is 10 .

At first we verify the solution obtained by comparing the proposed PGD based method to high fidelity Monte Carlo simulations. Next we comment upon the rate of convergence of the PGD representation. Furthermore we investigate the behavior of the basis functions $\boldsymbol{\Phi}(\cdot)$.

\section{A. Verification of Proposed Methodology}

To verify the solutions obtained using the proposed PGD based technique we compare the results with high fidelity
Monte Carlo (MC) simulations. The MC simulations are done by first randomly selecting 5000 points from the domain of $\Delta$. It is ensured that further addition of points did not change the expected value of the states significantly. We then solve the minimum expectation LQR problem to obtain the value of the gain $\boldsymbol{K}$. Details on how to solve a minimum expectation LQR problem using MC simulation has been omitted here and can be found in Ref. [14].

For the system in (28), the gain value obtained from MC simulations is given by $\boldsymbol{K}=[-16.30,-10.23]$. The gain obtained from the proposed PGD based technique is $\boldsymbol{K}=$ $[-16.86,-10.59]$. The percentage error in the solutions of PGD based method, assuming high fidelity MC simulation to generate actual solution is $[3.32 \%, 3.40 \%]$. It can be seen that the gain values obtained from the PGD based methodology are in close agreement with that obtained from high fidelity MC simulations. Furthermore, the closed loop eigenvalues obtained from MC simulations are $\boldsymbol{\lambda}_{c l}=-1.98 \pm \mathrm{i} 1.10$, and that obtained from PGD based method is $\boldsymbol{\lambda}_{c l}=-1.98 \pm$ i 1.11. Hence, it can be said that for the current F-16 based dynamical system, the outputs obtained from solving the minimum expectation $\mathrm{LQR}$ problem using the proposed PGD based method, matches that obtained from high fidelity MC simulation, upto an acceptable level of accuracy. The total time taken to run $5000 \mathrm{MC}$ samples is $10.2 s$ and total time taken for PGD based method is 8.1s. The simulations were run in a single Intel ${ }^{\circledR}$ Pentium ${ }^{\circledR}$ processor. Hence it can be said that to achieve similar accuracy PGD based method takes less time than MC.

\section{B. Rate of Convergence}

To investigate the rate of convergence, we observe the error term $e$ as given in Algorithm 11 The error $e$ is a measure of the contribution of an enrichment couple towards the PGD sum. We say that the PGD expansion has converged when the error $e$ is less than a $E_{t h}$. Figure 1 shows the variation of $e$ with the addition of enrichment couples. It can be seen that the error $e$ is minimized to a small value $\left(\sim 10^{-4}\right)$, rapidly. This indicates that the rate of convergence of the PGD based algorithm is almost exponential. However, it is observed that the decrease in $e$ is not monotonic, with an increase in the $5^{\text {th }}$ iteration. Although the actual reason of the increase in $e$ has not been investigated here, it can be attributed to the global nature of the PGD based algorithm [22]. Investigating the details about the convergence of PGD based algorithm is a topic of our future research.

\section{Investigating the PGD Basis Functions}

Figure 2 shows the first five $\left\{\Phi_{i}\right\}_{i=1}^{5}$ obtained for the short period F-16 dynamics. We discretize the $\Delta$ direction into 100 points distributed uniformly. The $\Phi_{i} \mathrm{~s}$ are then evaluated at those points. It can be observed that the basis functions exhibit a polynomial like behavior, with $\Phi_{1}$ resembling a constant function, $\Phi_{2}$ linear etc. Hence it can be said that for the current application, the states can be represented as linear combination of polynomials in $\boldsymbol{\Delta}$. Formal theoretical investigation about the smoothness and continuity of the 


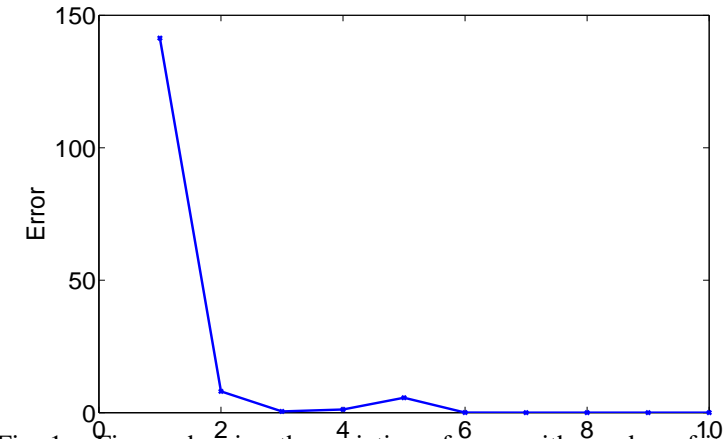

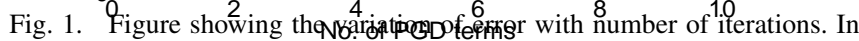
each iteration an enrichment couple is added.

basis functions has not been conducted here and is a subject of the author's future research.

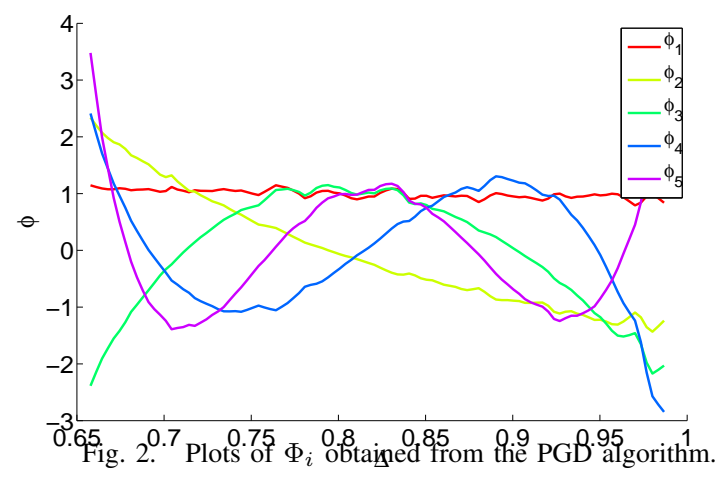

\section{CONCLUSIONS}

This work proposes a novel methodology of model order reduction for systems with parametric uncertainty using proper generalized decomposition. We consider a LTI with parametric uncertainties where the states are represented using PGD. The state feedback gain is found by solving a minimum expectation LQR.

The PGD based method is applied to control a F-16 aircraft with an uncertain parameter. We consider short period dynamics of the F-16 aircraft linearlized at trim values. The control objective is to maintain the aircraft at trim values.

It is observed that the proposed PGD based method can solve for the gain with sufficient accuracy, and achieve the desired control objective. Also it is found that the incremental error in adding an enrichment couple to the PGD sum decreases almost exponentially. This implies that the PGD can approximate the states of the current application fairly accurately, with a few number of terms.

In future we propose to extend the PGD based technique to control of nonlinear systems. Moreover a formal proof of convergence and comparison with other model reduction techniques is a topic of our future research.

\section{REFERENCES}

[1] S. Wiggins and M. Golubitsky, Introduction to applied nonlinear dynamical systems and chaos. Springer, 1990, vol. 2.
[2] T. Hull, W. Enright, B. Fellen, and A. Sedgwick, "Comparing numerical methods for ordinary differential equations," SIAM Journal on Numerical Analysis, vol. 9, no. 4, pp. 603-637, 1972.

[3] J. Guckenheimer and P. Holmes, Nonlinear oscillations, dynamical systems, and bifurcations of vector fields. New York Springer Verlag, 1983, vol. 42

[4] F. Chinesta, P. Ladeveze, and E. Cueto, "A short review on model order reduction based on proper generalized decomposition," Archives of Computational Methods in Engineering, vol. 18, no. 4, pp. 395-404, 2011.

[5] Y. Liang, H. Lee, S. Lim, W. Lin, K. Lee, and C. Wu, "Proper orthogonal decomposition and its applications-Part I: Theory," Journal of Sound and Vibration, vol. 252, no. 3, pp. 527-544, 2002.

[6] P. Dutta, A. Halder, and R. Bhattacharya, "Nonlinear filtering with transfer operator," in American Control Conference (ACC), 2013. IEEE, 2013, pp. 3069-3074.

[7] P. Dutta and R. Bhattacharya, "Nonlinear estimation of hypersonic state trajectories in bayesian framework with polynomial chaos," Journal of guidance, control, and dynamics, vol. 33, no. 6, pp. 1765$1778,2010$.

[8] E. Pruliere, F. Chinesta, and A. Ammar, "On the deterministic solution of multidimensional parametric models using the proper generalized decomposition," Mathematics and Computers in Simulation, vol. 81, no. 4, pp. 791-810, 2010.

[9] K. Takahashi, K. Yugi, K. Hashimoto, Y. Yamada, C. J. Pickett, and M. Tomita, "Computational challenges in cell simulation: a software engineering approach," Intelligent Systems, IEEE, vol. 17, no. 5, pp. 64-71, 2002

[10] A. Nouy, "A generalized spectral decomposition technique to solve a class of linear stochastic partial differential equations," Computer Methods in Applied Mechanics and Engineering, vol. 196, no. 45, pp. 4521-4537, 2007.

[11] M. Chevreuil and A. Nouy, "Model order reduction based on proper generalized decomposition for the propagation of uncertainties in structural dynamics," International Journal for Numerical Methods in Engineering, vol. 89, no. 2, pp. 241-268, 2012.

[12] D. González, F. Masson, F. Poulhaon, A. Leygue, E. Cueto, and F. Chinesta, "Proper generalized decomposition based dynamic data driven inverse identification," Mathematics and Computers in Simulation, vol. 82, no. 9, pp. 1677-1695, 2012.

[13] C. Ghnatios, F. Masson, A. Huerta, A. Leygue, E. Cueto, and F. Chinesta, "Proper generalized decomposition based dynamic datadriven control of thermal processes," Computer Methods in Applied Mechanics and Engineering, vol. 213, pp. 29-41, 2012.

[14] J. Fisher and R. Bhattacharya, "Linear quadratic regulation of systems with stochastic parameter uncertainties," Automatica, vol. 45 , no. 12 , pp. 2831-2841, 2009.

[15] F. Chinesta, A. Ammar, and E. Cueto, "Recent advances and new challenges in the use of the proper generalized decomposition for solving multidimensional models," Archives of Computational methods in Engineering, vol. 17, no. 4, pp. 327-350, 2010.

[16] J. Brewer, "Kronecker products and matrix calculus in system theory," IEEE Transactions on circuits and systems, vol. 25 , no. 9, pp. 772 781, 1978.

[17] A. E. Bryson, Applied optimal control: optimization, estimation and control. CRC Press, 1975.

[18] A. Nouy, "Generalized spectral decomposition method for solving stochastic finite element equations: invariant subspace problem and dedicated algorithms," Computer Methods in Applied Mechanics and Engineering, vol. 197, no. 51, pp. 4718-4736, 2008.

[19] J. W. Daniel, W. B. Gragg, L. Kaufman, and G. Stewart, "Reorthogonalization and stable algorithms for updating the Gram-Schmidt factorization," Mathematics of Computation, vol. 30, no. 136, pp. 772 795, 1976.

[20] Å. Björck, "Numerics of Gram-Schmidt orthogonalization," Linear Algebra and Its Applications, vol. 197, pp. 297-316, 1994.

[21] A. Young, C. Cao, N. Hovakimyan, and E. Lavretsky, "An adaptive approach to nonaffine control design for aircraft applications," in AIAA Guidance, Navigation, and Control Conference and Exhibit, Keystone, CO, USA, no. AIAA 2006-6343, 2006.

[22] A. Falcó and A. Nouy, "Proper generalized decomposition for nonlinear convex problems in tensor Banach spaces," Numerische Mathematik, vol. 121, no. 3, pp. 503-530, 2012. 